\title{
Simultaneous Localization and Tracking via Real-time Nonparametric Belief Propagation
}

\author{
Vladimir Savic and Henk Wymeersch
}

\section{Linköping University Post Print}

\section{Tweet}

N.B.: When citing this work, cite the original article.

C2013 IEEE. Personal use of this material is permitted. However, permission to reprint/republish this material for advertising or promotional purposes or for creating new collective works for resale or redistribution to servers or lists, or to reuse any copyrighted component of this work in other works must be obtained from the IEEE.

Original Publication:

Vladimir Savic and Henk Wymeersch, Simultaneous Localization and Tracking via Real-time Nonparametric Belief Propagation, 2013, EIII International Conference on Acoustics, Speech, and Signal Processing (ICASSP), 2013

38th International Conference on Acoustics, Speech, and Signal Processing (ICASSP 2013), 26-31 May 2013, Vancouver, Canada.

Postprint available at: Linköping University Electronic Press http://urn.kb.se/resolve?urn=urn:nbn:se:liu:diva-90015 


\title{
SIMULTANEOUS LOCALIZATION AND TRACKING VIA REAL-TIME NONPARAMETRIC BELIEF PROPAGATION
}

\author{
Vladimir Savic ${ }^{1}$ and Henk Wymeersch ${ }^{2}$ \\ ${ }^{1}$ Dept. of Electrical Engineering (ISY), Linköping University, Linköping, Sweden, Email: vladimir.savic@liu.se \\ ${ }^{2}$ Dept. of Signals and Systems, Chalmers University of Technology, Gothenburg, Sweden, Email: henkw@ chalmers.se
}

\begin{abstract}
Target tracking in wireless sensor networks is traditionally achieved by localization and tracking (LAT), where the sensors are first localized, and in a later stage the target is tracked. This approach is sub-optimal since the sensor-target observations are not used to refine the position estimates of the sensors. In contrast, simultaneous localization and tracking (SLAT) uses these observations to track the target while simultaneously localizing the sensors. In this paper, we propose a novel centralized SLAT method based on real-time nonparametric belief propagation, which has nearly the same complexity and the same communication cost as LAT, and can provide both sensors' and target's estimated distributions in non-Gaussian form.
\end{abstract}

Index Terms - Simultaneous localization and tracking, nonparametric belief propagation, wireless sensor networks

\section{INTRODUCTION}

Tracking a (passive) target in wireless sensor networks (WSN) requires position knowledge of each sensor, which can be achieved either by manually placing the sensors on predefined positions or equipping them with GPS receivers [1-4]. However, these approaches are typically impractical or too costly, so one alternative is to apply a WSN localization algorithm to locate all sensors. Then, these estimated locations, along with sensor-target observations, are used to track the target. This approach, referred to as localization and tracking (LAT) [5], is sub-optimal since the sensor-target observations (e.g., distance measurements) are not used to refine the sensor positions online. In contrast to this approach, simultaneous localization and tracking (SLAT), introduced in [6], uses these observations to track the target while simultaneously localizing the sensors, but typically with significantly higher complexity than LAT. In this paper, we propose a novel centralized SLAT method based on real-time nonparametric belief propagation $(N B P)$ which has nearly the same complexity and the same communication cost as LAT. This method, referred to as NBP-SLAT, can provide both sensors' and target's estimated distributions in non-Gaussian form.

\section{RELATION TO PRIOR WORK}

In the recent state-of-the-art, there are few important methods for SLAT [6-9]. In [6], the authors propose a Bayesian filtering approach to update a joint probability density functions (PDF) over the sensor positions, the target track, and the calibration parameters of

This work is supported by the Swedish Foundation for Strategic Research (SSF) and ELLIIT; Swedish Research Council (VR), under grant no. 2010-5889; and the European Research Council, under grant COOPNET No. 258418 . the network. To avoid huge computational complexity of this approach, Laplace's method has been used to approximate all PDFs with Gaussians. Since this approach estimates directly the joint PDF over all sensors, its complexity grows exponentially with the number of sensors. In [7], the authors propose distributed variational Bayesian filtering to find posterior marginal PDFs over the sensor positions and the target track. A variational approach, based on mean-field method, is used to factorize the joint PDF, which is then approximated with a Gaussian distribution. An extended Kalman filter $(\mathrm{EKF})$ is used in [8] to track the target while recursive maximum likelihood (ML) and expectation maximization (EM) determine the point estimates of the sensors' positions. A fully scalable distributed implementation is provided via message passing based on consensus propagation. Finally, in [9], authors propose a sequential Monte Carlo (SMC) approach to approximate the PDF of the target track, while they use EM and gradient descent to estimate the static parameters and the positions of the sensors, respectively. Therefore, the estimated PDFs of the tracks (not necessarily Gaussian) are available, but only the point estimates of the sensor positions.

None of the these methods are capable to provide all the characteristics of NBP-SLAT. Most importantly, we note that none of them can provide sensors' posterior PDFs in non-Gaussian form. Since [6] and [7] approximate posterior PDFs with a Gaussian distribution, and [8] and [9] with a point estimate (Dirac delta impulse), it will lead to a suboptimal approximation in case of non-linear models and/or non-Gaussian measurements. This is especially a serious problem in non-rigid graphs, in which the posterior PDFs of a subset of the sensors is multi-modal [10].

\section{PROBLEM FORMULATION}

We consider $N_{s}$ sensors with fixed 2D position $\mathbf{z}_{n}, n=1,2, \ldots, N_{s}$, scattered randomly in a planar region, and one target, with $2 \mathrm{D}$ position $\mathbf{x}_{t}$, at time $t\left(t=1,2, \ldots, N_{T}\right)$, moving through this region. A subset of the sensors (called anchors) can have perfect a priori position information. Prior to the target's arrival, the sensors are assumed to have executed a Bayesian (cooperative) localization algorithm $[11,12]$, leading to distributions of the positions of every sensor, represented by particles. The target is passive (e.g., a vehicle or person), in the sense that it cannot perform computations, but it can periodically emit a signal (e.g., acoustic) which can be detected by a subset of sensors, with a sampling interval $T_{s}$. We assume that sensors sense the target if and only if it is within a distance $r$. Two sensors can communicate if and only if they are within distance $R$ from one another. Assuming that the radio of a node is much more powerful than its sensing devices [13], $R>r$. Following [10], more complex models can be easily incorporated. Finally, we assume there is a fusion center (e.g., an external device or one of the sen- 
sors), which collects the priors of the sensors' and target's positions, and the periodic measurements. The goal of the fusion center is to determine posterior marginal PDFs (referred to as the beliefs) of the target at each time instant $t$, and of each sensor position.

\section{PROPOSED METHOD: SLAT VIA REAL-TIME NBP}

\subsection{Graphical model}

We first cast the estimation problem as a probabilistic graphical model. Since we only have pairwise (distance) measurements, we choose a Markov random field (MRF) model [14]. In a MRF, each vertex represents a random variable with an associated single-node potential (a local evidence), and each edge represents a pairwise potential (a likelihood function). An example of a MRF, with 3 sensors $(n, p$, and $q)$ and a target at three time instants $(t-1, t$, and $t+1$ ), is shown in Fig. 1. We introduce duplicate variables for the positions of the sensors at the different time instants, denoted by $\mathbf{z}_{n, t}$. Single and pairwise potentials ${ }^{1}$ are given by:

$$
\begin{gathered}
\psi_{t}\left(\mathbf{x}_{t}\right)=\left\{\begin{array}{l}
p_{0}\left(\mathbf{x}_{0}\right), \text { if } t=0 \\
1, \quad \text { otherwise }
\end{array}\right. \\
\psi_{n, t}\left(\mathbf{z}_{n, t}\right)=\left\{\begin{array}{l}
p_{n, 0}\left(\mathbf{z}_{n, 0}\right), \text { if } t=0 \\
1, \quad \text { otherwise }
\end{array}\right. \\
\psi_{t,(n, t)}\left(\mathbf{x}_{t}, \mathbf{z}_{n, t}\right)=p_{v}\left(d_{t,(n, t)}-\left\|\mathbf{x}_{t}-\mathbf{z}_{n, t}\right\|\right), \\
\psi_{t-1, t}\left(\mathbf{x}_{t-1}, \mathbf{x}_{t}\right)=p_{w}\left(\nu_{t-1}-\left\|\mathbf{x}_{t}-\mathbf{x}_{t-1}\right\| / T_{s}\right), \\
\psi_{(n, t-1),(n, t)}\left(\mathbf{z}_{n, t-1}, \mathbf{z}_{n, t}\right)=\delta\left(\mathbf{z}_{n, t}-\mathbf{z}_{n, t-1}\right),
\end{gathered}
$$

in which $p_{0}\left(\mathbf{x}_{0}\right)$ is the a priori distribution of the target's position, $p_{n, 0}\left(\mathbf{z}_{n, 0}\right)$ is the outcome of the initial localization algorithm, $d_{t,(n, t)}$ is the measured distance between the target at time $t$ and the sensor $n$ at time $t$ (distributed according to $\left.p_{v}(\cdot)\right), \nu_{t}$ is the measured speed of the target at time $t$ (distributed according to $p_{w}(\cdot)$ ), and $\delta(\cdot)$ is Dirac delta impulse, which enforces that the sensors are static.

The goal is to update the beliefs of the sensors' and target's positions in real-time. A tractable way to achieve it is by using a particle-based message passing method, such as NBP [10], but with the restrictions which ensure the real-time execution.

\subsection{Real-time belief propagation (BP)}

We focus on a single time slot $t(t>0)$, where at the beginning, the fusion center has available (i) the beliefs of the sensors' positions, based on all information up to time $t-1$, denoted by $M_{n, t-1}\left(\mathbf{z}_{n, t-1}\right)$; (ii) the belief of the target position, based on all information up to time $t-1$, denoted by $M_{t-1}\left(\mathbf{x}_{t-1}\right)$, as well as the single and pairwise potentials defined in previous section. Using standard BP (see [10, eqs. (8)-(9)]), we compute sensor-to-target and target-to-target messages:

$$
\begin{aligned}
& m_{(n, t) \rightarrow t}\left(\mathbf{x}_{t}\right)=\int \psi_{t,(n, t)}\left(\mathbf{x}_{t}, \mathbf{z}_{n, t}\right) \frac{M_{n, t}\left(\mathbf{z}_{n, t}\right)}{m_{t \rightarrow(n, t)}\left(\mathbf{z}_{n, t}\right)} d \mathbf{z}_{n, t}= \\
& =\int \psi_{t,(n, t)}\left(\mathbf{x}_{t}, \mathbf{z}_{n, t}\right) M_{n, t-1}\left(\mathbf{z}_{n, t}\right) d \mathbf{z}_{n, t} \\
& m_{t-1 \rightarrow t}\left(\mathbf{x}_{t}\right)=\int \psi_{t-1, t}\left(\mathbf{x}_{t-1}, \mathbf{x}_{t}\right) \frac{M_{t-1}\left(\mathbf{x}_{t-1}\right)}{m_{t \rightarrow t-1}\left(\mathbf{x}_{t-1}\right)} d \mathbf{x}_{t-1}= \\
& =\int \psi_{t-1, t}\left(\mathbf{x}_{t-1}, \mathbf{x}_{t}\right) M_{t-1}\left(\mathbf{x}_{t-1}\right) d \mathbf{x}_{t-1}
\end{aligned}
$$

\footnotetext{
${ }^{1}$ Note that the potentials and the messages are not necessarily normalized.
}

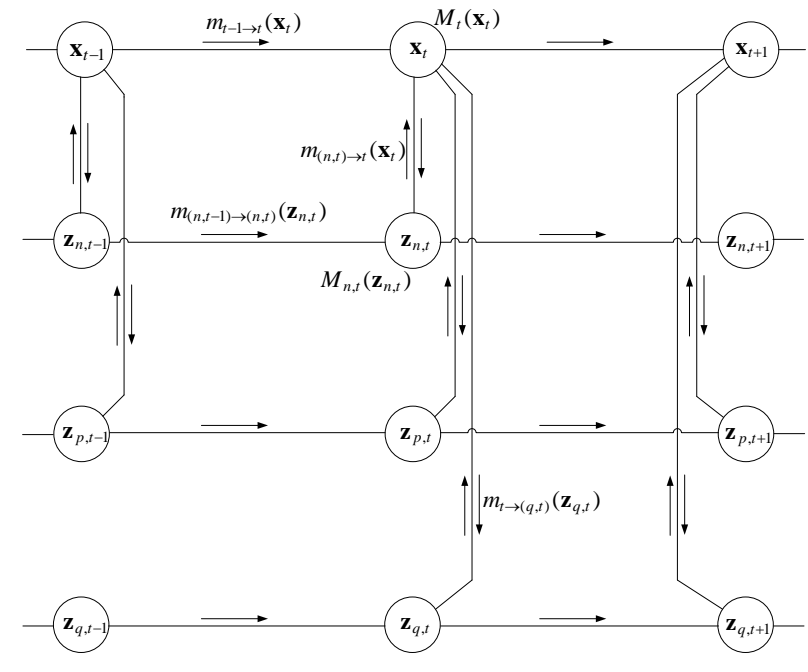

Fig. 1: Example of graphical model for online SLAT.

where we made the restriction to ensure real-time execution: $m_{t \rightarrow t-1}\left(\mathbf{x}_{t-1}\right)=1$. The target belief is given as the product of all received messages:

$$
M_{t}\left(\mathbf{x}_{t}\right) \propto m_{t-1 \rightarrow t}\left(\mathbf{x}_{t}\right) \prod_{n \in G_{t}^{s}} m_{(n, t) \rightarrow t}\left(\mathbf{x}_{t}\right)
$$

where $G_{t}^{s}$ is the set of sensors that can detect the target at time $t$.

Then, we compute the target-to-sensor and the sensor-to-sensor messages, respectively:

$$
\begin{aligned}
& m_{t \rightarrow(n, t)}\left(\mathbf{z}_{n, t}\right)=\int \psi_{t,(n, t)}\left(\mathbf{x}_{t}, \mathbf{z}_{n, t}\right) \frac{M_{t}\left(\mathbf{x}_{t}\right)}{m_{(n, t) \rightarrow t}\left(\mathbf{x}_{t}\right)} d \mathbf{x}_{t} \\
& m_{(n, t-1) \rightarrow(n, t)}\left(\mathbf{z}_{n, t}\right)= \\
= & \int \psi_{(n, t-1),(n, t)}\left(\mathbf{z}_{n, t-1}, \mathbf{z}_{n, t}\right) \frac{M_{n, t-1}\left(\mathbf{z}_{n, t-1}\right)}{m_{(n, t) \rightarrow(n, t-1)}\left(\mathbf{z}_{n, t-1}\right)} d \mathbf{z}_{n, t-1}= \\
= & M_{n, t-1}\left(\mathbf{z}_{n, t}\right)
\end{aligned}
$$

where we used (5) and made the following restriction to ensure realtime execution: $m_{(n, t) \rightarrow(n, t-1)}\left(\mathbf{z}_{n, t-1}\right)=1$. Finally, we update the belief of the sensors:

$$
M_{n, t}\left(\mathbf{z}_{n, t}\right) \propto\left\{\begin{array}{l}
M_{n, t-1}\left(\mathbf{z}_{n, t}\right) m_{t \rightarrow(n, t)}\left(\mathbf{z}_{n, t}\right), \text { if } n \in G_{t}^{s} \\
M_{n, t-1}\left(\mathbf{z}_{n, t}\right), \text { otherwise }
\end{array}\right.
$$

Remarks: Note that we have not included (online) inter-sensor measurements, which can provide additional information, but also significantly increase the complexity and latency. However, these links would be necessary if the sensors are mobile (in that case, the corresponding algorithm is cooperative SLAT (CoSLAT), proposed in [15]). Moreover, despite the presence of loops of the MRF, real-time BP does not schedule messages through those loops, so that the real-time beliefs are exact. ${ }^{2}$ Finally, we note that traditional

\footnotetext{
${ }^{2}$ An offline approach, which includes information from the future, would provide over-confident beliefs. In that case, reweighted variants of BP (e.g., $[11,12])$ should be used.
} 
LAT can be considered as a specific instance of SLAT, by restricting the target-to-sensors messages (i.e., instead of computing (9), we set $\left.m_{t \rightarrow(n, t)}\left(\mathbf{z}_{n, t}\right)=1\right)$.

\subsection{Real-time nonparametric belief propagation (NBP)}

Due to the nonlinearity and possible non-Gaussian measurement noise, parametric BP (where the beliefs and messages are computed using distributions belonging to parametric families) is undesirable. Therefore, we apply nonparametric approximation in which all the beliefs are represented with a weighted set of particles.

We assume we have available a set of $N_{p}$ weighted particles from the previous time instant: $\left\{W_{n, t-1}^{(j)}, \mathbf{Z}_{n, t-1}^{(j)}\right\},\left\{W_{t-1}^{(j)}, \mathbf{X}_{t-1}^{(j)}\right\}$ $\left(j=1,2 \ldots N_{p}\right)$ which represent the approximation of the beliefs $M_{n, t-1}\left(\mathbf{z}_{n, t-1}\right)$ and $M_{t-1}\left(\mathbf{x}_{t-1}\right)$, respectively. Our goal is to obtain the approximation of these beliefs at time $t$.

We first approximate the message (6), by shifting the particle $\mathbf{Z}_{n, t-1}^{(j)}$ in a random direction by an amount that represents the estimated distance between the sensor $n$ and the target at time $t$. Thus, the particles and weights are given by:

$$
\begin{gathered}
\mathbf{X}_{(n, t) \rightarrow t}^{(j)}=\mathbf{Z}_{n, t-1}^{(j)}+\left(d_{t,(n, t)}-v_{n, t}^{(j)}\right)\left[\cos \theta_{n, t}^{(j)} \sin \theta_{n, t}^{(j)}\right]^{T} \\
W_{(n, t) \rightarrow t}^{(j)}=W_{n, t-1}^{(j)}
\end{gathered}
$$

where $v_{n, t}^{(j)} \sim p_{v}(\cdot)$ and $\theta_{n, t}^{(j)} \sim$ Uniform $[0,2 \pi)$. We can also determine a continuous approximation of (6), via Monte Carlo (MC) integration. Using (5) and (6), we find:

$$
m_{(n, t) \rightarrow t}^{\mathrm{MC}}\left(\mathbf{x}_{t}\right)=\sum_{j} \psi_{t,(n, t)}\left(\mathbf{x}_{t}, \mathbf{Z}_{n, t-1}^{(j)}\right) W_{n, t-1}^{(j)}
$$

In case of the message from an anchor, we do not draw the particles, since the parametric form of the message is simply given by $m_{(a, t) \rightarrow t}^{\mathrm{MC}}\left(\mathbf{x}_{t}\right)=m_{(a, t) \rightarrow t}\left(\mathbf{x}_{t}\right)=\psi_{t,(a, t)}\left(\mathbf{x}_{t}, \mathbf{Z}_{a, t}\right)$ where $\mathbf{Z}_{a, t}$ is the known position of the anchor $a$ at time $t$. Similarly, we can approximate (7) in particle and continuous form:

$$
\begin{gathered}
\mathbf{X}_{t-1 \rightarrow t}^{(j)}=\mathbf{X}_{t-1}^{(j)}+\left(\nu_{t-1}-w_{t-1}^{(j)}\right) T_{s}\left[\cos \theta_{t-1, t}^{(j)} \sin \theta_{t-1, t}^{(j)}\right]^{T} \\
W_{t-1 \rightarrow t}^{(j)}=W_{t-1}^{(j)} \\
m_{t-1 \rightarrow t}^{\mathrm{MC}}\left(\mathbf{x}_{t}\right)=\sum_{j} \psi_{t-1, t}\left(\mathbf{x}_{t}, \mathbf{X}_{t-1}^{(j)}\right) W_{t-1}^{(j)}
\end{gathered}
$$

where $w_{t-1}^{(j)} \sim p_{w}(\cdot)$ and $\theta_{t-1, t}^{(j)} \sim$ Uniform $[0,2 \pi)$.

Now, we will approximate (8), which would require us to draw particles from the product of the messages. Since this is in general intractable, we use a proposal distribution, the sum of Gaussian mixtures with reference particles (RPs), and then reweight the particles. This approach, called mixture importance sampling with RPs (MIS-RP) [16], is extension of standard MIS [10]. We first create a collection of $\left(\left|G_{t}^{s,-a}\right|+1+\delta_{\mathrm{RP}}\right) N_{p}$ weighted particles by taking all particles from each incoming message excluding anchors (i.e., $\mathbf{X}_{t-1 \rightarrow t}^{(j)}$ and $\mathbf{X}_{(n, t) \rightarrow t}^{(j)}, \forall n \in G_{t}^{s,-a}, j=1,2 \ldots N_{p}$; where $G_{t}^{s,-a}$ is the set of non-anchors which can detect the target at time $t$ ) and adding a small number of uniformly distributed particles (RPs) over whole deployment area. Here, $\delta_{\mathrm{RP}}$ is the percentage of reference particles. These RPs are especially useful in case of outliers [17]. Then, we draw $N_{p}$ particles $\left(\mathbf{X}_{t}^{(j)}\right)$ from this collection, and com- pute their weights as importance ratio:

$$
W_{t}^{(j)}=\frac{m_{t-1 \rightarrow t}^{\mathrm{MC}}\left(\mathbf{X}_{t}^{(j)}\right) \prod_{n \in G_{t}^{s}} m_{(n, t) \rightarrow t}^{\mathrm{MC}}\left(\mathbf{X}_{t}^{(j)}\right)}{m_{t-1 \rightarrow t}^{\mathrm{MC}}\left(\mathbf{X}_{t}^{(j)}\right)+\sum_{n \in G_{t}^{s,-a}} m_{(n, t) \rightarrow t}^{\mathrm{MC}}\left(\mathbf{X}_{t}^{(j)}\right)+\delta_{\mathrm{RP}}}
$$

We finally normalize the weights $W_{t}^{(j)}=W_{t}^{(j)} / \sum_{j^{\prime}} W_{t}^{\left(j^{\prime}\right)}$ and have an approximation of (8). We will now approximate (9) as

$$
m_{t \rightarrow(n, t)}^{\mathrm{MC}}\left(\mathbf{z}_{n}\right)=\sum_{j} \psi_{t,(n, t)}\left(\mathbf{X}_{t}^{(j)}, \mathbf{z}_{n, t}\right) \frac{W_{t}^{(j)}}{m_{(n, t) \rightarrow t}^{\mathrm{MC}}\left(\mathbf{X}_{t}^{(j)}\right)}
$$

which is computed only if $n$ is not an anchor (otherwise, it is not necessary to send this message). We finally update the weighted particles of the sensor beliefs (11) as:

$$
\begin{gathered}
\mathbf{Z}_{n, t}^{(j)}=\mathbf{Z}_{n, t-1}^{(j)} \\
W_{n, t}^{(j)}=\left\{\begin{array}{l}
W_{n, t-1}^{(j)} m_{t \rightarrow(n, t)}^{\mathrm{MC}}\left(\mathbf{Z}_{n, t}^{(j)}\right), \quad \text { if } t \in G_{n}^{\tau} \\
W_{n, t-1}^{(j)}, \quad \text { otherwise }
\end{array}\right.
\end{gathered}
$$

These weights are then normalized to sum up to one. Note that in principle, it is again possible to use MIS-RP, but since there is maximum one incoming message this is not necessary.

Finally, we can compute the estimated positions of the sensors and the target as minimum-mean-square-error (MMSE) estimate, i.e., $\mathbf{x}_{t}^{\text {est }}=\sum_{j} W_{t}^{(j)} \mathbf{X}_{t}^{(j)}$, and $\mathbf{z}_{n, t}^{\text {est }}=\sum_{j} W_{n, t}^{(j)} \mathbf{Z}_{n, t}^{(j)}$. A wide variety of other estimates can be also found (e.g., mode, variance, probability that target/sensor is within some critical area). Regarding complexity, taking that the computation of (18) is dominant, both SLAT and LAT need $\mathcal{O}\left(\left|G_{t}^{s}\right| N_{p}^{2}\right)$ operations per time slot.

Remarks: There is a potential problem with the previous procedure. Some of the calculated weights can be much larger then the rest, which means that the particle-based estimate will be dominated by the influence of a few (or just one) particles. This problem, known as sample depletion [18], can significantly increase the estimated error. To avoid this problem, we use resampling with replacement $[10,18]$ for the particle-based approximation of the sensor's and the target's beliefs (after (18) and (21), respectively), in which we draw $N_{p}$ particles with uniform weights from the weighted set of particles. Although the resampling step reduces the sample depletion, it introduces another problem, sample impoverishment, in which most of the particles (now with the same weights) collapse to a single point. This problem, which is severe in case of small measurement noise, we solve by adding additional noise to the particles (called, jitter) $[18,19]$.

\section{NUMERICAL EXAMPLE}

We consider a network with 3 sensors in a $100 \mathrm{~m}$ by $100 \mathrm{~m}$ area, and one target moving with constant speed $30 \mathrm{~m} / \mathrm{s}$. The initial position of the target $(t=1)$ and two sensors are perfectly known. We set the sensing radius to $r=45 \mathrm{~m}$, the communication radius to $R=50 \mathrm{~m}$, the number of particles to $N_{p}=1000$, and percentage of RPs to $\delta_{\mathrm{RP}}=0.1$. The sampling interval is set to $T_{s}=1 \mathrm{~s}$, and there are only 3 discrete intervals. We assume that the measured distance is distributed according to Gaussian distribution with the mean corresponding to true value and standard deviation $\sigma_{d}=2 \mathrm{~m}$. Finally, to ensure the fair comparison, we provide the same seed for all random variables (in this case, the measurements and the particles). 


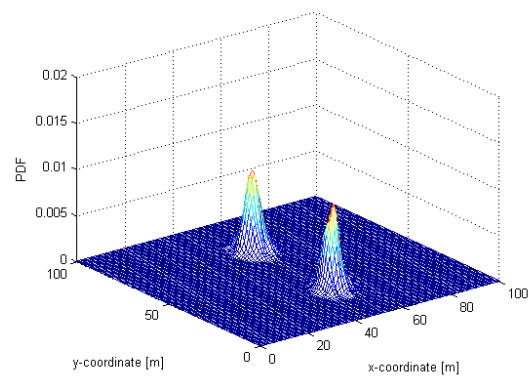

(a)

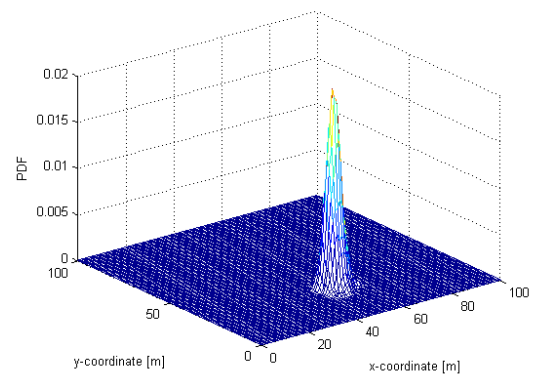

(d)

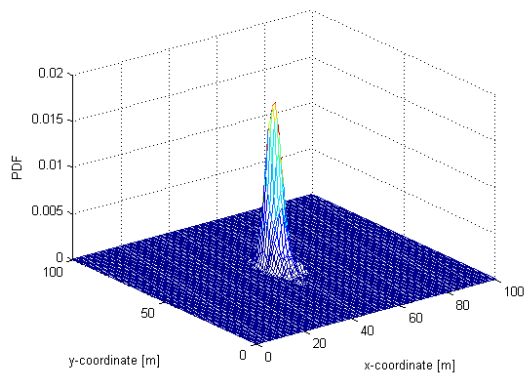

(b)

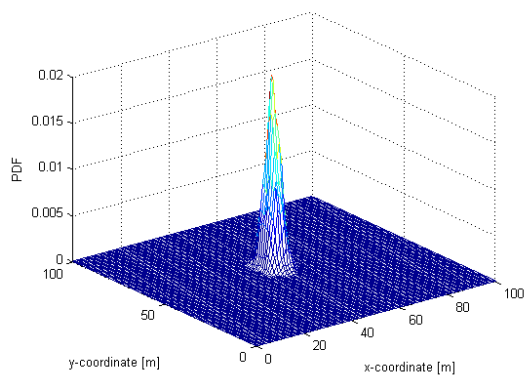

(e)

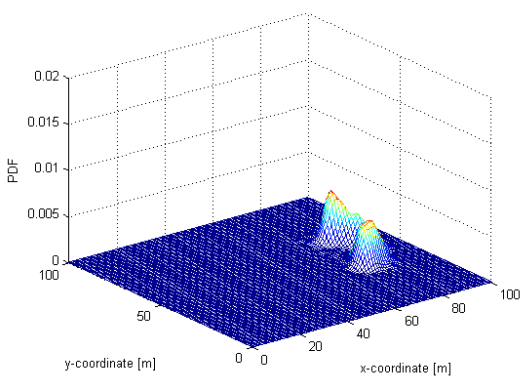

(c)

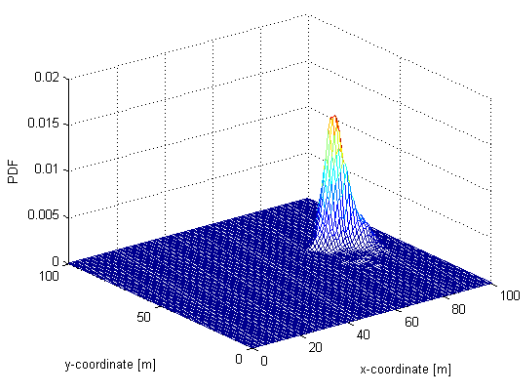

(f)

Fig. 2: The estimated beliefs of the position of: (a) sensor 2 (NBP-LAT), (b) target at $t=2$ (NBP-LAT), (c) target at $t=3$ (NBP-LAT), (d) sensor 2 (NBP-SLAT), (e) target at $t=2$ (NBP-SLAT), and (f) target at $t=3$ (NBP-SLAT).

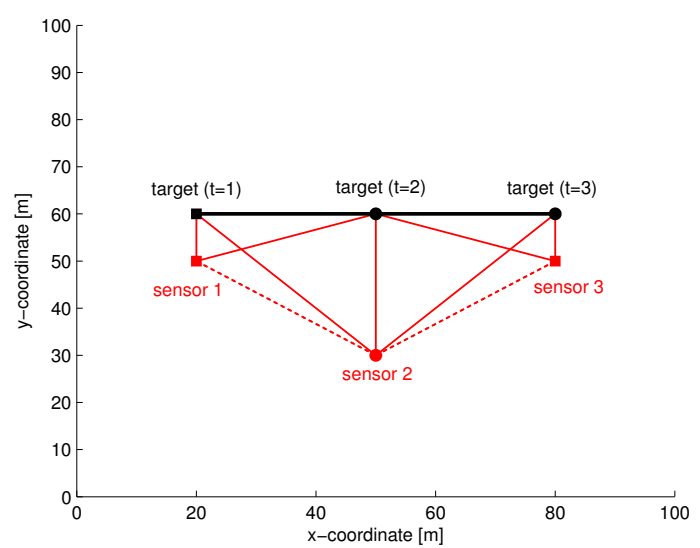

Fig. 3: A 3-node network and a target at 3 time instants. The known positions of the sensors/target are marked by squares, and unknown with circles. The dashed edges are only used to obtain the prior of sensor 2 , and all others for online SLAT.

This scenario, which corresponds to the graphical model in Fig. 1, is illustrated in Fig. 3.

Our goal is to compare the estimates of NBP-SLAT with NBPLAT. The beliefs ${ }^{3}$ of sensor 2 , and the target at times $t=2,3$ are shown in Fig. 2. As we can see, sensor 2 has only two sensors as

\footnotetext{
${ }^{3}$ Given weighted particles, the beliefs are visualized using kernel density estimation with optimal bandwidth selection [20].
}

neighbours, so its belief (found a priori) is bimodal (Fig. 2a). This belief, along with the known positions of other two sensors, is used to track the target at times $t=2,3$. As a result, the belief of target at time $t=2$ (Fig. 2b) is more informative than belief at time $t=3$ (Fig. 2c), since it has three neighbors with perfectly known position. In contract to this approach, in NBP-SLAT, sensor 2 also uses the information from the target to improve its position, so its final belief is unimodal. This improved belief is used to track the target (again, along with known positions of other two sensors), so the target estimates are also improved (especially, at time $t=3$ ).

In total, root-mean-square error is reduced from $13.73 \mathrm{~m}$ to 2.86 $\mathrm{m}$ while the execution time increased only $9 \%$. Similar results are obtained for other examples not included in this paper. Moreover, as mentioned earlier, the communication cost of both NBP-SLAT and NBP-LAT is exactly the same. Therefore, NBP-SLAT should be always used instead of NBP-LAT.

\section{CONCLUSIONS}

We presented a novel SLAT method based on real-time NBP, with the following characteristics: i) it has nearly the same complexity as LAT, ii) its communication cost is exactly the same as LAT, and iii) can provide both sensors and target's beliefs in non-Gaussian form. According to our results, NBP-SLAT is significantly more accurate than NBP-LAT, especially if the sensors' beliefs are multimodal. Our future work will focus on the performance analysis of the NBP-SLAT in large-scale WSNs, comparison with other stateof-the-art methods, and distributed implementation. 


\section{REFERENCES}

[1] S. Farahmand, S. I. Roumeliotis, and G. B. Giannakis, "Setmembership constrained particle filter: Distributed adaptation for sensor networks," IEEE Transactions on Signal Processing, vol. 59, pp. 4122-4138, Sept. 2011.

[2] O. Hlinka, O. Sluciak, F. Hlawatsch, P. M. Djuric, and M. Rupp, "Distributed gaussian particle filtering using likelihood consensus," in Proc. of IEEE Int. Conf. on Acoustics, Speech and Signal Processing (ICASSP), pp. 3756-3759, May 2011.

[3] D. Ustebay, M. Coates, and M. Rabbat, "Distributed auxiliary particle filters using selective gossip," in Proc. of IEEE Int. Conf. on Acoustics, Speech and Signal Processing (ICASSP), pp. 3296-3299, May 2011.

[4] V. Savic, H. Wymeersch, and S. Zazo, "Distributed target tracking based on belief propagation consensus," in Proc. of the 20th European Signal Processing Conference (EUSIPCO), Aug. 2012.

[5] E. L. Souza, E. F. Nakamura, and H. A. de Oliveira, "On the performance of target tracking algorithms using actual localization systems for wireless sensor networks," in Proc. of the ACM Intl. Conf. on Modeling, Analysis and Simulation of Wireless and Mobile Systems (MSWiM), pp. 418-423, Oct. 2009.

[6] C. Taylor, A. Rahimi, J. Bachrach, H. Shrobe, and A. Grue, "Simultaneous localization, calibration, and tracking in an ad hoc sensor network," in Proc. of IEEE/ACM Int. Conf. on Information Processing in Sensor Networks (IPSN), pp. 27-33, April 2006.

[7] J. Teng, H. Snoussi, C. Richard, and R. Zhou, "Distributed variational filtering for simultaneous sensor localization and target tracking in wireless sensor networks," IEEE Transactions on Vehicular Technology, vol. 61, pp. $2305-2318$, June 2012.

[8] N. Kantas, S. Singh, and A. Doucet, "Distributed maximum likelihood for simultaneous self-localization and tracking in sensor networks," IEEE Transactions on Signal Processing, vol. 60, pp. 5038-5047, Oct. 2012.

[9] X. Chen, A. Edelstein, Y. Li, M. Coates, M. Rabbat, and A. Men, "Sequential Monte Carlo for simultaneous passive device-free tracking and sensor localization using received signal strength measurements," in Proc. of IEEE/ACM Int. Conf. on Information Processing in Sensor Networks (IPSN), pp. 342-353, April 2011.
[10] A. T. Ihler, J. W. I. Fisher, R. L. Moses, and A. S. Willsky, "Nonparametric belief propagation for self-localization of sensor networks," IEEE Journal on Selected Areas in Communications, vol. 23, pp. 809819, April 2005.

[11] H. Wymeersch, F. Penna, and V. Savic, "Uniformly reweighted belief propagation for estimation and detection in wireless networks," IEEE Transactions on Wireless Communications, vol. 11, pp. 1587-1595, April 2012.

[12] V. Savic, H. Wymeersch, F. Penna, and S. Zazo, "Optimized edge appearance probability for cooperative localization based on treereweighted nonparametric belief propagation," in Proc. of IEEE Int. Conf. on Acoustics, Speech and Signal Processing (ICASSP), pp. 30283031, May 2011.

[13] B. Jiang and B. Ravindran, "Completely distributed particle filters for target tracking in sensor networks," in Proc. of IEEE Int. Parallel \& Distributed Processing Symp., pp. 334-344, May 2011.

[14] M. Wainwright and M. Jordan, Graphical models, exponential families, and variational inference. Now Publishers Inc., 2008.

[15] F. Meyer, E. Riegler, O. Hlinka, and F. Hlawatsch, "Simultaneous distributed sensor self-localization and target tracking using belief propagation and likelihood consensus," in Proc. of Asilomar Conference on Signals, Systems, and Computers, Nov. 2012.

[16] V. Savic and S. Zazo, "Cooperative localization in mobile networks using nonparametric variants of belief propagation," Ad Hoc Networks, vol. 11, pp. 138-150, Jan. 2013.

[17] D. Fox, W. Burgard, F. Dellaert, and S. Thrun, "Monte Carlo localization: Efficient position estimation for mobile robots," in Proc. of the National Conference on Artificial Intelligence, July 1999.

[18] M. S. Arulampalam, S. Maskell, N. G. Gordon, and T. Clapp, "A tutorial on particle filters for online nonlinear/non-Gaussian Bayesian tracking," IEEE Transactions on Signal Processing, vol. 50, pp. 174188, Feb. 2002

[19] F. Gustafsson, F. Gunnarsson, N. Bergman, U. Forsell, J. Jansson, R. Karlsson, and P. J. Nordlund, "Particle filters for positioning, navigation and tracking," IEEE Transactions on Signal Processing, vol. 50, pp. 425-437, Feb. 2002.

[20] Z. I. Botev, "A novel nonparametric density estimator," tech. rep., The University of Queensland, Australia, 2006. 